\title{
It's under control: Top-down search strategies can override attentional capture
}

\author{
ANDREW B. LEBER and HOWARD E. EGETH \\ Johns Hopkins University, Baltimore, Maryland
}

\begin{abstract}
Bacon and Egeth (1994) proposed that observed instances of attentional capture by feature singletons (e.g., color) were the result of a salience-based strategy adopted by subjects (singleton detection mode) and, thus, were not automatic. They showed that subjects could override capture by adopting strategies based on searching for specific target features (feature search mode). However, Theeuwes (2004) has recently argued that Bacon and Egeth's results arose from experimental confounds. He elaborated a model in which attentional capture must be expected when salient distractors fall within a spatial window of attention. According to Theeuwes's (2004) model, there exist two essential criteria for examining stimulus-driven capture. First, search latencies cannot increase with display size, since the search must be parallel; second, the salience of the irrelevant distractor must not be compromised by characteristics of the search display. Contrary to the predictions of Theeuwes's (2004) model, we provide evidence that involuntary capture can be overridden when both of these criteria are met. Our results are consistent with Bacon and Egeth's proposal.
\end{abstract}

In a recent article entitled "Top-Down Search Strategies Cannot Override Attentional Capture," Theeuwes (2004; see also 1991a, 1992) argued against the popular notion that featurally salient information in the visual scene can be ignored when it does not match the attentional set of the observer. He claimed, rather, that the selection of sensory information is always granted preferentially to the most salient stimulus within a spatially defined window of attention. As a result, when subjects are engaged in parallel processing, salient distractors should automatically capture attention.

At face value, it may seem difficult to reconcile this account with a great deal of research on attention. Visual search studies have, for decades, shown that the manner in which stimuli are prioritized for selection is dramatically influenced by the observer's strategic goals (e.g., Green \& Anderson, 1956), and virtually all recent models of attention incorporate a major component of top-down control (e.g., Wolfe, 1994). Furthermore, numerous studies, employing various experimental designs, have shown that irrelevant, featurally salient distractors do not automatically impair subjects' performance on visual search tasks (e.g., Folk, Leber, \& Egeth, 2002; Folk, Remington, \& Johnston, 1992; Lamy \& Tsal, 1999; Theeuwes, 1990; Yantis \& Egeth, 1999). These studies have been widely interpreted to support the contingent capture hypothesis

This research was supported in part by Grant 2001-G-020 from the TSA. We thank Angus Gellatly, Bryan Burnham, and an anonymous reviewer for thoughtful comments and suggestions on a previous version of the manuscript. We also thank James Drakakis, Lauren Hanlon, and Ivy Rosenthal for assistance with data collection. Correspondence concerning this article should be addressed to A. B. Leber, Department of Psychology, Yale University, P.O. Box 208205, New Haven, CT 065208205 (e-mail: andrew.leber@yale.edu). advanced by Folk and colleagues (e.g., Folk et al., 2002; Folk et al., 1992), which states that the degree to which a salient stimulus involuntarily captures attention is dependent on the degree to which that item matches the observer's attentional set.

\section{Inside the Window: A Clarification of Theeuwes's Position}

Do the myriad findings of selectivity in the literature counter Theeuwes's (2004) assertion that feature singletons automatically capture attention? Perhaps not. Critically, Theeuwes (2004) made a distinction between objects that fall inside a window of attention-much like a spotlight (see Posner, Snyder, \& Davidson, 1980) - and those that do not. Within the window, objects compete in parallel for selection, and this competition is based purely on salience, so that the most salient item wins the highest processing priority (e.g., Theeuwes, 1991a, 1992, 2004). Objects outside the window, however, do not necessarily compete for selection and can, therefore, be ignored (see Theeuwes, 1990, 1991b; Theeuwes \& Burger, 1998). Thus, Theeuwes's brand of automaticity applies not to all stimuli impinging upon the retina but, rather, only to those falling inside an attentional window.

One essential facet of Theeuwes's (2004) model is that the attentional window can vary in size much like the zoom lens proposed by Eriksen and Yeh (1985). For example, in a simple pop-out search, observers could open a broad window-encompassing the entire visual field - to search for a target in parallel. Here, the most salient item in the display should win the competition. Accordingly, Theeuwes (e.g., 1991a, 1992, 2004) has observed sizable distractor interference in studies in which the target was a singleton. However, in contrast to opening a broad window, observers may elect to open a small window when 
they know with certainty where a target will appear. Here, salient distractors outside the window (i.e., in nontarget locations) should fail to capture attention, and empirical findings have supported this prediction (Theeuwes, 1991b; Yantis \& Jonides, 1990; but see Folk et al., 2002).

One further relevant stipulation of Theeuwes's (2004) model is that the window can move dynamically about the visual field. For example, when observers engage in a difficult search task, they could open a small windowencompassing, say, one or two items at a time - and move it serially around the display until the target is found. At any given moment, a salience-based competition occurs only inside the window. Therefore, if a salient distractor is present in the display, it need not capture attention to a noticeable degree, because (1) it should not affect stimulus prioritization when it is outside the window, (2) it should fall inside the window only on a fraction of the trials, since the search may be terminated before the window is moved over the target, and (3) when the distractor does fall inside the window, it may not cause interference if the window is sufficiently small (i.e., if the window encompasses only one item at a time, there is never a salience-based competition for the distractor to win). Consequently, a moving window account can be agnostic as to whether salient distractors impact stimulus prioritization during serial search (e.g., Theeuwes, 1990, 2004; Theeuwes \& Burger, 1998). Since the signature of serial search is an increase in response time (RT) as a function of display size, one seeking to test Theeuwes's model must be wary of data containing positive search slopes. ${ }^{1}$ The critical question is, "can search proceed in parallel and yet, at the same time, be selective toward the task relevant dimension?" (Theeuwes, 1991a, p. 184).

Theeuwes's (2004) window provides a vantage point on the attentional capture literature that may be surprisingly parsimonious. By convention, following Yantis and Jonides (1984), many studies have deliberately incorporated difficult search tasks as part of a methodology of assessing attentional capture. In fact, most studies to date in which it has been concluded that singletons did not automatically capture attention featured difficult search tasks. These yielded positive search slopes in baseline conditions (e.g., Jonides \& Yantis, 1988; Lamy \& Tsal, 1999; Theeuwes, 1990; Todd \& Kramer, 1994; Yantis \& Egeth, 1999). Furthermore, other studies reporting negligible capture effects either did not manipulate display size (e.g., Folk et al., 1992; Turatto \& Galfano, 2001) or did not employ methods allowing one to readily ascertain whether parallel search could have occurred (Folk et al., 2002). As a result, Theeuwes's model, which requires evidence of parallel search, remains largely untested.

\section{An Alternative to Windows: Strategic Modes of Processing}

Although the attentional window account promises a reconciliation of seemingly contrary findings in the literature regarding feature-based selectivity, alternatives exist. Bacon and Egeth (1994) raised the possibility that observers can be selective to the task-relevant feature - even during parallel search - but do not always demonstrate such capabilities. Following Pashler (1988), Bacon and Egeth proposed that observers can often engage visual search tasks with one of two basic search strategies. The singleton detection mode is based purely on local salience; subjects using this mode assign the highest priority for selection to the most salient information in the display (e.g., singletons). In contrast, the feature search mode capitalizes on observers' abilities to impose top-down selectivity; subjects using this mode should not be susceptible to capture by stimuli not matching the attentional set.

Bacon and Egeth (1994) noted that both modes might have been available to the subjects in Theeuwes's (1991a, 1992) earlier experiments, in which the task was to search for a singleton target (e.g., an outline circle among outline diamonds; see Figure 1A). Although it is conceivable that feature search mode could have been used when the defining target feature was consistent across trials (Theeuwes, 1992), the subjects may not have been inclined to use it,
A

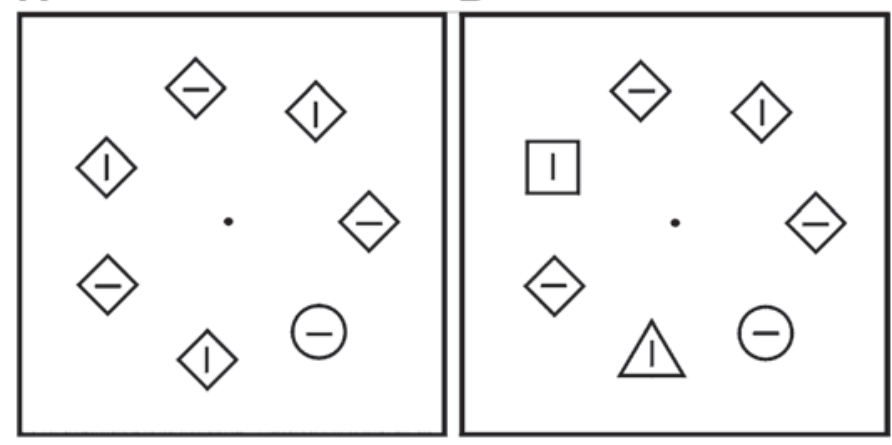

Figure 1. (A) Representation of the stimuli used by Theeuwes (1991a; see also Bacon \& Egeth, 1994, Experiment 1). The subjects reported the orientation of the line segment inside the circle. (B) Representation of Bacon and Egeth's (Experiment 3) modification to Theeuwes's displays. Because of the shape heterogeneity, the target was not the only item in the display that was unique with respect to form. 
since they had the option to prioritize salient items in the display via the singleton detection mode.

Bacon and Egeth (1994) sought evidence for the feature search mode by creating conditions that discouraged subjects from using the singleton detection mode. Consider, for example, their third experiment, modeled after Theeuwes's (1991a, 1992) paradigm. Instead of asking the subjects to always search for a singleton circle among diamonds, Bacon and Egeth introduced shape heterogeneity to some displays, by replacing some nontarget diamonds with unique shapes (e.g., triangles and squares; see Figure 1B). Specifically, one diamond was replaced on one third of the trials, whereas two diamonds were replaced on another third of the trials. On the remaining third of the trials, however, no diamonds were replaced, thus keeping the target a singleton; these trials were critical, since they were identical to those used by Theeuwes (1991a, 1992; see also Bacon \& Egeth, Experiment 1). The three trial types were mixed within blocks, ensuring that the subjects could not expect a singleton target on every trial and that a singleton mode would be ineffective. Bacon and Egeth found that color singleton distractors failed to influence RT on any of the three trial types-including those in which the target was a singleton. These results indicated to them that the subjects tapped their ability to be selective and adopted a block-wide strategy of feature search mode, thus overriding capture by the salient color singleton.

\section{Recent Developments in the Debate}

Do Bacon and Egeth's (1994) results lay the issue to rest? One possible weakness in their data, pointed out by Theeuwes (2004), is that the RTs did increase significantly as a function of display size, opening the possibility that serial search was used. However, the slopes were actually quite shallow-less than $5 \mathrm{msec} /$ item on the critical third of the trials in which the target was a singleton (Bacon \& Egeth, 1994, Experiment 3). Such a rate suggests that any attentional window would need to be large enough to encompass a majority, if not the entirety, of the items at each moment. Given such a large window, Theeuwes's model appears to predict that Bacon and Egeth should have observed at least some interference. Nonetheless, we acknowledge the pertinence of what we term the serial search criticism.

A second concern Theeuwes (2004) raised about Bacon and Egeth's (1994) results is that the methods employed to revoke the singleton status of the targets (e.g., by introducing shape heterogeneity into the displays) not only reduced the salience of the target, but also reduced the salience of the color distractors. This reduced salience criticism draws support from the findings that visual salience is tightly linked to the homogeneity of objects in the search array (e.g., Duncan \& Humphreys, 1989; Nothdurft, 1993). However, one critical aspect of Bacon and Egeth's design that Theeuwes may have overlooked is that one third of the trials contained singleton targets, in stimulus conditions modeled after his original experiments. On these particular trials, the reduced salience criticism appears unwarranted. (One might argue, however, that the remaining two thirds of the trials not containing a shape singleton encouraged the observers to use a serial search on all the trials, thus raising again the serial search criticism.)

Theeuwes (2004) sought to test Bacon and Egeth's (1994) account, while averting the criticisms he raised. Like Bacon and Egeth (1994, Experiment 3), he presented no more than three unique forms (the target and two additional nontarget shapes), but he used larger display sizes (12 and 20 items, as opposed to the initial displays of 5-9 items). These stimuli endowed the unique shapes (and the singleton distractor) with higher local salience, because the background was more homogeneous (see Duncan \& Humphreys, 1989; Nothdurft, 1993). The results stood in contrast to those of Bacon and Egeth; the color singleton created significant interference, and there was no effect of display size. Furthermore, in a control experiment, he reduced the display sizes to 5 and 9 and essentially replicated Bacon and Egeth's results: no interference and positive slopes. Theeuwes (2004) interpreted these results to mean that when search is parallel and the distractor is sufficiently salient, capture is automatic.

Unfortunately, although Theeuwes's (2004) recent study avoided some problems, it may have created others. Particularly, increasing the display size - and consequently, the homogeneity - to increase salience was antithetical to Bacon and Egeth's (1994) initial intention of discouraging the use of singleton detection mode. That is, since each unique form became more salient at the larger display sizes, a salience-driven search (i.e., the singleton mode) was now a suitable means of finding the target. Consider that four conspicuous items (distractor, target, and two additional unique forms) now popped out in the displays; subjects using a salience-driven search would have to search only within this set of 4 items (out of an average display size of 16) to find the target. Such a motivational incentive was not available to Bacon and Egeth's subjects.

\section{A New Approach}

A proper test of Theeuwes's model would need to determine whether an irrelevant singleton interferes with performance when (1) search is parallel and (2) distractor salience is not compromised by such factors as display heterogeneity. We felt that perhaps the best way to achieve this was by simply reverting to the stimuli Theeuwes had used in his earlier studies (e.g., 1992) - specifically, the same singleton target on every trial. Subjects in these conditions have repeatedly demonstrated parallel search (e.g., Bacon \& Egeth, 1994, Experiment 1; Theeuwes, 1992), and it would be difficult to dispute the high salience of the singleton distractors. However, we needed to coax subjects to use feature search mode, since past research has suggested a preference for singleton detection. To this end, we capitalized on our recent finding that past experience plays a significant role in the implementation of attentional set (Leber \& Egeth, in press). This work showed that subjects tend to persist with a preestablished set, even when stimulus conditions change.

In the present experiment we preceded a test phase of 480 trials like those in Theeuwes (1992) with a train- 
ing phase of 480 trials designed to require the use of the feature search mode. In this training phase, feature group subjects were exposed to stimuli much like those in Bacon and Egeth's (1994) third experiment; they had to search for a circle among heterogeneous nontarget shapes (diamonds, squares, and triangles). We expected that the subjects would use the feature search mode in this circumstance, as did the subjects in Bacon and Egeth's third experiment.

Would these subjects persist in using the feature search mode into the test phase, which contained trials like those in Theeuwes (1992), in such a manner that the color singletons could be ignored? If observed in the context of parallel search, where the distractor is shown to be salient, such a finding would violate Theeuwes's window account.

We took one additional step to ensure that the singleton distractor was salient during the test phase by including a singleton group, in which the subjects searched for an unpredictable target (circle, triangle, or diamond) among homogeneous nontarget shapes (all squares) during the training phase. Subsequently, these subjects were exposed to the same test phase as the feature group; given that these subjects would likely persist in searching for singletons, distractor interference would be probable, and such interference would confirm the color singleton's inherent salience.

\section{METHOD}

\section{Subjects}

Thirty Johns Hopkins undergraduates with self-reported normal or corrected-to-normal visual acuity and normal color vision participated in a session lasting approximately $50 \mathrm{~min}$.

\begin{abstract}
Materials
Stimuli were generated with a personal computer and displayed on a black background on a 19-in. VGA monitor. The subjects used a chinrest placed at a viewing distance of approximately $57 \mathrm{~cm}$. A white fixation cross, measuring $0.5^{\circ}$ in width and $0.5^{\circ}$ in height, with a stroke of $0.05^{\circ}$, was positioned in the center of the screen. Search displays consisted of five or nine outline shapes equally spaced around the circumference of an imaginary circle with a radius of $3^{\circ}$ that was centered at fixation. The outline shapes, all drawn with a stroke of $0.1^{\circ}$, could be a circle (diameter of $\left.1.5^{\circ}\right)$, a square $\left(1.3^{\circ}\right.$ per side), a diamond (a square rotated $45^{\circ}$ ), or an equilateral triangle (pointing upward, at $1.5^{\circ}$ per side). The outline shapes were colored green, with the exception of the singleton distractor, which was colored red, when present. Either a vertical or a horizontal line segment $\left(0.5^{\circ}\right.$ in length, with a stroke of $\left.0.05^{\circ}\right)$, colored white, was centered inside each shape.
\end{abstract}

\section{Design}

Half of the subjects were assigned to the singleton group, whereas the remaining subjects were assigned to the feature group.

Training phase. Depending on group assignment, the subjects were exposed to one of two display types. (1) The subjects in the singleton group searched for a target of varied shape (circle, diamond, or triangle, each presented on a random one third of the trials) among nontargets that were all identical in shape (squares). (2) The subjects in the feature group searched for a target of consistent shape (always a circle) among nontargets that were heterogeneous in shape. These nontarget items always included one diamond and one triangle; the remaining shapes were squares. For both groups of subjects, spatial positioning of the items was randomized.

Test phase. All the subjects were treated identically in this phase. The targets were consistent in shape (always a circle) among nontargets that were all identical in shape (squares). Spatial positioning of the target was randomized.

Within-subjects variables. For both groups, and within each phase, three independent variables were manipulated within subjects to determine the stimulus characteristics on each trial: distractor condition (two levels), display size (two levels), and target orientation (two levels). This yielded eight unique conditions, each of which was presented 60 times in each phase, for a total of 480 trials per phase; presentation order was randomized within each phase. The variables are described as follows.

Distractor condition. In distractor-absent trials (half of all the trials), all the outline shapes were colored green. In distractor-present trials (the remaining trials), one nontarget shape was colored red, and the remaining shapes were colored green.

Display size. Half of the trials contained five search items, whereas the remaining trials contained nine.

Target orientation. The orientation of the line segment inside the target was vertical on half of the trials and horizontal on the remaining trials. Each of the line segments appearing inside nontarget shapes was selected randomly and independently on each trial.

\section{Procedure}

During the training phase, the singleton group subjects were instructed to search for the item possessing a unique shape, whereas the feature group subjects were instructed to search for the circle. In the test phase, all the subjects were instructed to search for the circle for the remainder of the experiment. Upon finding the target shape, the subjects were asked to report the orientation of the line segment inside it, by pressing 1 on the numberpad of a standard keyboard for vertical bars and 2 for horizontal bars. They were encouraged to respond as quickly as possible, while keeping errors to a minimum.

The experiment consisted of 24 task familiarization trials, followed by 480 training phase trials and 480 test phase trials, including periodic breaks. Trials began with the presentation of fixation for $500 \mathrm{msec}$. Next, the search display was presented for $2,000 \mathrm{msec}$, or until response. Trials in which the subjects failed to respond within $2,000 \mathrm{msec}$ were counted as errors. On these trials and on trials in which the subjects entered incorrect responses, a feedback tone was presented for $500 \mathrm{msec}$, followed by a 1,000-msec blank, yielding a $1,500-\mathrm{msec}$ penalty. Successive trials were temporally separated by a $500-\mathrm{msec}$ intertrial interval.

\section{RESULTS AND DISCUSSION}

RT data were trimmed - separately for the training and test phases - on correct response trials at 3.5 standard deviations above the mean, for each subject, within each distractor condition and display size. Approximately $1.2 \%$ of the correct response trials for each subject were removed by this procedure.

\section{Training Phase}

Mean training phase RTs and error rates are shown in Table 1. RTs were entered into a 2 (group assignment) $\times$ 2 (display size) $\times 2$ (distractor condition) ANOVA. The results showed a significant main effect of group assignment $\left[F(1,28)=18.296, M S_{\mathrm{e}}=581,846.788, p<.00001\right]$, reflecting slower performance in the singleton group than in the feature group; this is consistent with research showing that search for unknown singleton targets is slower than search for known features (e.g., Bravo \& Nakayama, 1992). 
Table 1

Training Phase: Mean Response Times and Error Rates With Display Sizes of Five and Nine

\begin{tabular}{|c|c|c|c|c|c|c|c|c|}
\hline \multirow[b]{3}{*}{ Condition } & \multicolumn{4}{|c|}{ Response Time (msec) } & \multicolumn{4}{|c|}{ Error Rate $(\%)$} \\
\hline & \multicolumn{2}{|c|}{$\begin{array}{l}\text { Singleton } \\
\text { Group }\end{array}$} & \multicolumn{2}{|c|}{$\begin{array}{l}\text { Feature } \\
\text { Group }\end{array}$} & \multicolumn{2}{|c|}{$\begin{array}{l}\text { Singleton } \\
\text { Group }\end{array}$} & \multicolumn{2}{|c|}{$\begin{array}{l}\text { Feature } \\
\text { Group }\end{array}$} \\
\hline & 5 & 9 & 5 & 9 & 5 & 9 & 5 & 9 \\
\hline Distractor & 910 & 912 & 711 & 725 & 2.1 & 2.1 & 3.5 & 3.7 \\
\hline No distractor & 817 & 800 & 713 & 726 & 2.5 & 2.5 & 3.7 & 3.7 \\
\hline
\end{tabular}

A main effect of display size was not observed $(F<1)$. However, this was qualified by a display size $\times$ group interaction $\left[F(1,28)=7.625, M S_{\mathrm{e}}=2,991.732, p<.02\right]$, reflecting greater search slopes in the feature group $(3.4 \mathrm{msec} /$ item) than in the singleton group $(0.3 \mathrm{msec} / \mathrm{item})$. Indeed, planned comparisons revealed a significant display size effect in the feature group $[t(14)=3.073, p<.01]$ but no effect in the singleton group $[t(14)=1.191$, n.s.]. We note that the significant, although modest, mean slope in the feature group was similar to the $5.3-\mathrm{msec} /$ item slope found by Bacon and Egeth (1994, Experiment 3) in their physically similar condition containing three unique forms.

Distractor condition yielded a significant main effect $\left[F(1,28)=68.278, M S_{\mathrm{e}}=76,178.562, p<.00001\right]$ and interacted significantly with group assignment $[F(1,28)=$ $\left.72.613, M S_{\mathrm{e}}=81,014.957, p<.00001\right]$ as a result of large interference in the singleton group. Planned comparisons revealed negligible interference in the feature group [ $2 \mathrm{msec} ; t(14)=0.732$, n.s.] and large interference in the singleton group $[t(14)=9.537, p<.00001]$. No other interactions reached significance.

Error rates in both training groups were small and did not vary as a function of distractor condition or display size (see Table 1). Taken together, the training phase data suggest that the two groups of observers adopted the strategies encouraged by our experimental manipulations; conceivably, the respective groups could persist in using these distinct strategies into the test phase.

\section{Test Phase}

The remaining analyses focused on performance in the test phase, in which all the subjects were treated identically. Mean RTs are plotted, by group assignment, in Figure 2 and were entered into a 2 (group assignment) $\times 2$ (display size) $\times 2$ (distractor condition) ANOVA. The main effect of distractor condition was significant $[F(1,28)=$ $\left.20.559, M S_{\mathrm{e}}=255.298, p<.0001\right]$; this was qualified by a significant group assignment $\times$ distractor condition interaction $\left[F(1,28)=6.095, M S_{\mathrm{e}}=255.298, p=.02\right]$, reflecting larger interference in the singleton group than in the feature group. Indeed, planned contrasts revealed that the observers in the singleton group suffered significant distractor interference $[20 \mathrm{msec} ; t(14)=5.828, p<$ $.001],{ }^{2}$ whereas the observers in the feature group did not incur a significant cost $[6 \mathrm{msec} ; t(14)=1.308, \mathrm{n} . \mathrm{s}$.$] .$

A main effect of display size was not observed $\left[F(1,28)=1.768, M S_{\mathrm{e}}=412.181\right.$, n.s. $]$, nor did it interact with group assignment $(F<1)$, suggesting parallel search in both groups. Furthermore, to be reasonably assured that the data did not reflect positive search slopes, we computed $95 \%$ confidence intervals around the display size functions for each group: The slopes for the singleton and feature groups were $-0.8 \pm 1.7 \mathrm{msec} /$ item and $-1.0 \pm$ $2.4 \mathrm{msec} /$ item, respectively, affirming that the slopes were reliably flat.

Although an examination of the means reveals that the feature group was nominally slower than the singleton
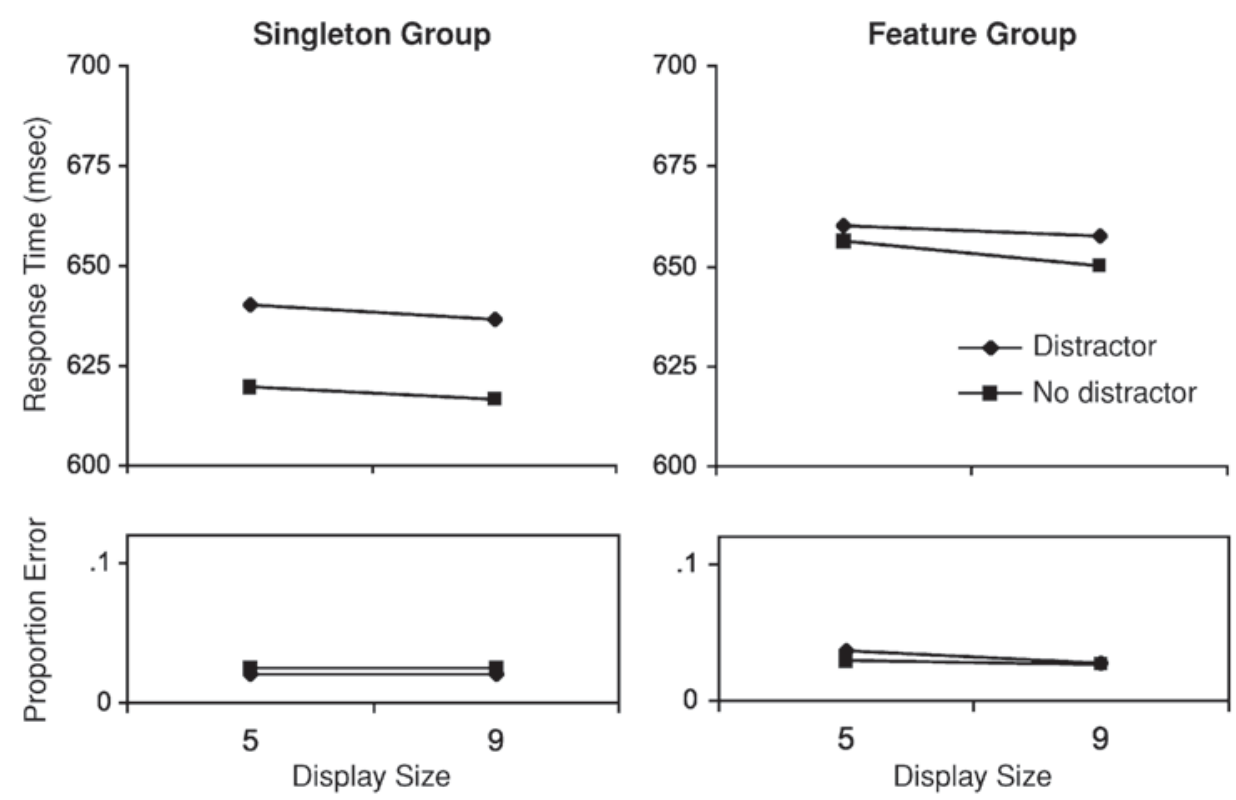

Figure 2. Mean response times (in milliseconds) and error rates in the test phase as a function of distractor condition and display size, plotted by training phase group assignment. 
group, the main effect of group assignment was not significant $[F(1,28)=1.887 \text {, n.s. }]^{3}$ The remaining interactions failed to reach significance $\left(F_{\mathrm{S}}<1\right)$.

Error rates were small and did not vary significantly as a function of distractor condition, display size, or training group (below 4\% in all conditions; see Figure 2).

The test phase data are unambiguous and appear to contradict the account of Theeuwes (e.g., 2004). First, the search slopes were not positive, indicating that the subjects did not perform a serial — or even partly serial — search. Second, the distractor was salient, since it was potent enough to cause substantial interference for the subjects initially assigned to the singleton group. Finally, the subjects initially assigned to the feature group, who presumably persisted with their preestablished set, successfully avoided interference from the distractor. Taken together, these results are difficult to reconcile with the claim that the most salient item in the display automatically draws attention during parallel search. Rather, it would appear that, as Bacon and Egeth (1994) and others (e.g., Folk et al., 1992; Jonides \& Yantis, 1988) have claimed, subjects possess the ability to exert top-down control to avert involuntary capture by known-to-be-irrelevant, albeit salient, feature singletons.

\section{REFERENCES}

Bacon, W. F., \& Egeth, H. E. (1994). Overriding stimulus-driven attentional capture. Perception \& Psychophysics, 55, 485-496.

Bravo, M. J., \& NaKaYAMa, K. (1992). The role of attention in different visual-search tasks. Perception \& Psychophysics, 51, 465-472.

Duncan, J., \& Humphreys, G. W. (1989). Visual search and stimulus similarity. Psychological Review, 96, 433-458.

EgETH, H. (1977). Attention and preattention. In G. H. Bower (Ed.), The psychology of learning and motivation (Vol. 11, pp. 277-320). New York: Academic Press.

Eriksen, C. W., \& YeH, Y.-Y. (1985). Allocation of attention in the visual field. Journal of Experimental Psychology: Human Perception \& Performance, 11, 583-597.

Folk, C. L., Leber, A. B., \& Egeth, H. E. (2002). Made you blink! Contingent attentional capture produces a spatial blink. Perception \& Psychophysics, 64, 741-753.

Folk, C. L., \& Remington, R. W. (1998). Selectivity in distraction by irrelevant featural singletons: Evidence for two forms of attentional capture. Journal of Experimental Psychology: Human Perception \& Performance, 24, 847-858.

Folk, C. L., Remington, R. W., \& Johnston, J. C. (1992). Involuntary covert orienting is contingent on attentional control settings. Journal of Experimental Psychology: Human Perception \& Performance, 18, 1030-1044.

Green, B. F., \& Anderson, L. K. (1956). Color coding in a visual search task. Journal of Experimental Psychology, 51, 19-24.

JoNides, J., \& YANTIS, S. (1988). Uniqueness of abrupt visual onset in capturing attention. Perception \& Psychophysics, 43, 346-354.

Lamy, D., Carmel, T., Egeth, H. E., \& Leber, A. B. (in press). Effects of search mode and intertrial priming on singleton search. Perception \& Psychophysics.

LAmY, D., \& TsaL, Y. (1999). A salient distractor does not disrupt conjunction search. Psychonomic Bulletin \& Review, 6, 93-98.

Leber, A. B., \& Egeth, H. E. (in press). Attention on autopilot: Past experience and attentional set. Visual Cognition.

NothduRfT, H.-C. (1993). The role of features in preattentive vision: Comparison of orientation, motion and color cues. Vision Research, 33, 1937-1958.

PAShler, H. (1988). Cross-dimensional interaction and texture segregation. Perception \& Psychophysics, 43, 307-318.
Pinto, Y., Olivers, C. N. L., \& Theeuwes, J. (2005). Target uncertainty does not lead to more distraction by singletons: Intertrial priming does. Perception \& Psychophysics, 67, 1354-1361.

Posner, M. I., SNyder, C. R., \& Davidson, B. J. (1980). Attention and the detection of signals. Journal of Experimental Psychology: General, 109, 160-174.

Teichner, W. H., \& Krebs, M. J. (1974). Laws of visual choice reaction time. Psychological Review, 81, 75-98.

Theeuwes, J. (1990). Perceptual selectivity is task dependent: Evidence from selective search. Acta Psychologica, 74, 81-99.

Theeuwes, J. (1991a). Cross-dimensional perceptual selectivity. Perception \& Psychophysics, 50, 184-193.

Theeuwes, J. (1991b). Exogenous and endogenous control of attention: The effect of visual onsets and offsets. Perception \& Psychophysics, 49, 83-90.

Theeuwes, J. (1992). Perceptual selectivity for color and form. Perception \& Psychophysics, 51, 599-606.

TheEuwes, J. (1996). Perceptual selectivity for color and form: On the nature of the interference effect. In A. F. Kramer, M. G. H. Coles, \& G. D. Logan (Eds.), Converging operations in the study of visual attention (pp. 297-314). Washington, DC: American Psychological Association.

TheEuwes, J. (2004). Top-down search strategies cannot override attentional capture. Psychonomic Bulletin \& Review, 11, 65-70.

Theeuwes, J., \& Burger, R. (1998). Attentional control during visual search: The effect of irrelevant singletons. Journal of Experimental Psychology: Human Perception \& Performance, 24, 1342-1353.

Todd, S., \& Kramer, A. F. (1994). Attentional misguidance in visual search. Perception \& Psychophysics, 56, 198-210.

Townsend, J. T. (1971). A note on the identifiability of parallel and serial processes. Perception \& Psychophysics, 10, 161-163.

Turatto, M., \& Galfano, G. (2001). Attentional capture by color without any relevant attentional set. Perception \& Psychophysics, 63, 286-297.

Wolfe, J. M. (1994). Guided Search 2.0: A revised model of visual search. Psychonomic Bulletin \& Review, 1, 202-238.

Yantis, S., \& EgETh, H. E. (1999). On the distinction between visual salience and stimulus-driven attentional capture. Journal of Experimental Psychology: Human Perception \& Performance, 25, 661-676.

Yantis, S., \& Jonides, J. (1984). Abrupt visual onsets and selective attention: Evidence from visual search. Journal of Experimental Psychology: Human Perception \& Performance, 10, 601-621.

Yantis, S., \& Jonides, J. (1990). Abrupt visual onsets and selective attention: Voluntary versus automatic allocation. Journal of Experimental Psychology: Human Perception \& Performance, 16, 121-134.

\section{NOTES}

1. Although positive RT $\times$ display size functions do not necessarily reflect serial search (e.g., Townsend, 1971), positive slopes allow the criticism that search may have been serial.

2 . The reader may notice that interference effects in the singleton group were markedly reduced between the training and the test phases. This reduction is partially the result of a gradual improvement in performance over the course of the test phase (e.g., interference fell to $68 \mathrm{msec}$ by the final 40 trials of the training phase). The remaining difference may indicate that some or all of the observers capitalized on their knowledge that the target feature no longer varied randomly in the test phase; thus, they may have abandoned a pure singleton detection mode (for recent evidence that subjects use top-down guidance in search for consistent target singletons, see Lamy, Carmel, Egeth, \& Leber, in press; but see Pinto, Olivers, \& Theeuwes, 2005).

One might question whether the observed interference in the singleton group resulted from shifts of spatial attention or solely from a central filtering cost (see Folk \& Remington, 1998). We examined this by comparing trials in which the distractor's line orientation matched (compatible) and did not match (incompatible) the reported target feature. The results were inconclusive: A 7-msec effect was found in the test phase $[t(14)=2.223, p<.05]$, but no such effect obtained during training, where distractor interference was greater $[-10 \mathrm{msec} ; t(14)=-1.309$, n.s.]. These results may have arisen because we used simple line seg- 
ments, rather than more complex stimuli; there is at least some basis for thinking that alphanumeric characters are compulsively encoded (e.g., Teichner \& Krebs, 1974), whereas line orientation may not be (e.g., Egeth, 1977). In accord with this possibility, Theeuwes (1996) reported significant compatibility effects, using near-identical displays with letters as the reported feature, rather than line segments. Given Theeuwes's (1996) results, we believe that spatial attention likely moved to the distractors, but our use of line stimuli limited the ability to measure this.

3. However, an unpaired two-tailed $t$ test, restricted to the distractorabsent condition only, approached significance, when uncorrected for post hoc comparisons $[t(28)=1.768, p<.1]$. In light of these marginal results, one could question whether the subjects were able to avoid - or conceal effects of - capture simply by responding less rapidly. This line of reasoning would predict that the more slowly a subject responds, the less interference will be observed. However, we found no correlation between distractor-absent RTs and interference effect in the feature group during the test phase $\left(r^{2}=.000\right.$, n.s. $)$. Indeed, the 7 fastest subjects, whose distractor-absent RTs were comparable to the singleton group's (617 msec), did not suffer significant interference [ $7 \mathrm{msec} ; t(6)=0.922$, n.s.].

(Manuscript received July 22, 2004;

revision accepted for publication May 8, 2005.) 